\title{
Voices of AYAO
}

$T$ HE "Voices of AYAO" feAture in each issue of JAYAO will have stories from real patients and survivors, in their own words. It is our hope that these illustrate the personal journeys and experiences of AYAs living through and surviving with cancer and its effects.

-Editors

\section{Jenee's Story}

Cancer survivors live in two worlds- "the now" and the "what if." Although we won the battle, the enemy at times is not too far behind, and there is always fear that it will return. The spectrum of emotions a survivor goes through ranges from amazement at beating the big " $\mathrm{C}$ " to one of pure loneliness. We learn to protect not only ourselves, but also the ones we love.

At 15, my world turned upside down when a misdiagnosed sprained knee led to a terrible fall down a flight of stairs at my school in Spain. I was immediately flown to the Mayo Clinic in Rochester, Minnesota, where tests confirmed my parents' worst fear-it was osteosarcoma. The orthopedic surgeon discussed two treatment options with us: amputate my leg or fuse the knee. This was a hard decision. In my mind both options meant a loss, as it was either no leg or a stiff leg with the possibility of recurrence ending in amputation. Confused and indecisive as to which way to go, the orthopedic surgeon brought an amputee to visit with me. This allowed me the opportunity to address some immediate and future concerns: "Can you drive?" "How do you buy shoes?" "Can people tell the difference when you're wearing clothes?" "Did you date?" "Are you married?" After talking to the amputee, I made the decision to amputate, feeling I could do more with a prosthesis than a fused knee. My parents were shocked, as they had assumed I would want to save my leg. But I felt empowered to make this difficult decision because the orthopedic team gave me the opportunity to ask questions and become educated about life as an amputee. Allowing me to have control over this loss helped me cope with the cancer. On January 10, 1985, my left leg was amputated above the knee, a decision I do not regret to this day. After the amputation, I was casted and fitted with a new prosthesis and a renewed life.

Before returning to Spain, CT scans declared me cancerfree, which meant no chemotherapy was needed. The plan was to undergo monthly CT scans to monitor the osteosarcoma. Shortly after, both lungs collapsed, one after the other, as the scans showed metastasis to both lungs with 13 nodules and a small lemon-sized mass hitting my spine near my heart. Treatment consisted of a year of chemotherapy, two lung surgeries to remove the nodules, and an insertion of six radiation seeds. Once again, I was declared cancer-free.

At 18, my fear was realized again. A tiny nodule was found on the wall of my heart, resulting in open-heart surgery and confirming a relapse of the osteosarcoma. It was then back to chemotherapy for another year and a new clinical trial consisting of VP16, ifosfamide, and mesna.

Five years later, at the age of 24, I finally became a survivor of my adolescent cancer. Through this long and arduous journey, I never felt alone. I was surrounded by an awesome pediatric multidisciplinary team consisting of oncologists, surgeons, nurses, social workers, child life specialists, and psychologists. They supported me each step of the way and made the experience comfortable.

Ten years later, at the age of 35, I graduated with a Masters in social work from the University of Southern California. I was ready to finally realize my dream of becoming a pediatric oncology social worker, and was hired by CHOC Children's. Merging both social work skills and personal experience, I knew I could help teens deal with the emotional and psychosocial implications of their cancers.

While feeling that life could not get any better and focused on my new career, I was once again blindsided. During a myomectomy procedure, a mass was discovered on my ovary. Tests confirmed that it was Stage $2 \mathrm{~B}$ ovarian cancer. I was only 38 and full of hope to one day have children of my own. But in less than 24 hours, I went from possible to never when the only treatment option available to me was a total hysterectomy followed by six weeks of chemotherapy. My body shot right into menopause.

The shock was intense. Things happened so fast that I had no time to think and felt the decision to remove my womb was stripped away from me. I was given little education about my options and had no opportunity to speak with a fellow survivor to help me process what was happening. I just wanted to give up and felt unsupported by the gynecology oncology team. In my mind, it was a routine surgery for them-did they care that they were permanently altering my life by forever taking away one of the most precious gifts I had as woman, the ability to give birth to a child? It was easier to overcome the loss of my leg than the loss of my ability to bear children. I felt empty and numb.

With the help of my pediatric oncologist and gynecologist, I was able to be heard and change to a new gynecology oncologist. I experienced neuropathy mid-treatment, which is especially problematic when you are an amputee with only one good leg to begin with. The numbness began hindering my quality of life and the fear of having another permanent loss in my life set in. I could not bear the loss of walking. This psychosocial issue, which I feel should have been recognized and discussed during treatment planning, was not addressed.

The losses between the osteosarcoma and the ovarian cancer were so different. As an amputee, I overcame many losses attached to having one leg, such as walking, driving, caring for myself, working, swimming, skiing, and performing ethnic 
dances, which gave me the ability to live a full and normal life. The losses associated with ovarian cancer are more difficult to overcome, as they are permanent, such as the feeling of emptiness because you have no uterus or ovaries and are unable to give life to your own flesh and blood. As an amputee, I feel I can still relate to an able-bodied person better than I can relate to someone with kids. On a daily basis, I am reminded of my dreams of being a mother in others.

But time is everyone's healer. I see a new opportunity and purpose in dedicating my efforts to improving the needs of the AYA group, my new baby! Each of us AYA survivors has something to give back in battling the big " $\mathrm{C}$ " by helping new patients through the emotions attached to life during and after cancer. We, the AYA survivors and patients, have a voice and need to say, "STOP! Hear what we are saying, know we are entitled to be emotionally supported, to be cared for as humans, and to have access to good healthcare fulfilling our atypical needs."

In my experience, there is a great disparity between the care of an adolescent and a young adult cancer patient. For example, when I was an adolescent and needed a central line, it was done under general anesthesia. As a young adult, the central line placement was done under local anesthesia. It was the most horrific experience-I felt the cut of my skin, the pressure of placing the portacath underneath my skin, and the pulling and tugging as the needle sewed me shut. This would never happen in the pediatric world, so why should this happen in the adult world? The standards of care should be the same. Regardless of age, the feelings and the pain do not change. One of my biggest issues is how young adults must tolerate and "suck it up" while adolescents can succumb to their feelings. Having experienced both sides, I felt more supported as an adolescent patient than as an adult one, and I wish I had been treated in the pediatric oncology ward.

I have found purpose in dedicating my efforts to address the unique psychosocial issues and needs of AYAs with cancer. As AYA survivors, we have to give back and serve as a light of hope for those fighting the battle. AYAs need to know they have a voice and the ability to advocate for themselves. They need to be educated, given all their options, and feel empowered to make decisions about their treatment. Their quality of care should not be compromised or any different from that in the pediatric world.

Living with cancer for the past 26 years has taught me a great many things, but most importantly, it has taught me to cherish every moment of life.

Jenee Areeckal, now 41 years old, is of Asian Indian decent and a proud survivor of osteosarcoma (17 years) and ovarian cancer (3 years). A pediatric oncology social worker at CHOC Children's, she is proud to be a "challenged athlete"-skiing, swimming, and recently taking up wheelchair tennis, including organizing a yearly wheelchair tennis clinic for her patients.

\section{Selma's Story}

"This unfortunate young woman has a newly diagnosed carcinoma of the breast. At her age, with a medical lesion, I am very worried..." Those are the words that appeared in my hospital chart following my first medical oncology consult. I was diagnosed with breast cancer in 1983 at age 28, though I first found the hard mass in my left breast months prior. Being diagnosed and simply being taken seriously as a young woman who could have breast cancer was one of the greatest challenges of my life.

This happened on the heels of my mother's death from ovarian cancer just 10 weeks after her diagnosis. I could not ignore the firm mass that did not behave like a cyst and knew there was something seriously wrong. I first saw my gynecologist, who had also seen my mother, and surely, I thought, would be extra cautious with me. But he told me I was "too young for breast cancer."

Next was a radiologist-surely at least a mammogram was in order. But this was still a time with little understanding of its limitations in young adult women with denser breasts; I will never forget how he held my films to the light box, reminded me I was too young for breast cancer and said, "See, there's nothing wrong with you. Go home, you're being neurotic." Those words were core to my survival. While I felt very alone in that moment, I somehow knew my life hung on those words- I'd rather be neurotic then dead.

Another gynecologist was also unresponsive to my plea to biopsy that mass, which now was causing a cramping sensation that felt like a spasm in my chest wall, even as I was told "cancer does not hurt." I thought a general surgeon might help me and recalled a surgical resident I used to know. He was now in private practice; I went to his office without an appointment and sat in the waiting room. Surprised by my unexpected visit, he too felt it was unlikely my breast mass was malignant and wanted to continue waiting and watching. But finally I met him in a Santa Monica emergency room. I knew he decided to excise it for my peace of mind and was still certain it was benign. However, the short procedure became lengthy and a second physician came to assist. He peeled back the disposable blue towel to say hello and covered me again. It was suffocating, and the procedure, with nothing more than a valium, was painful. I remember the silence when the preliminary pathology came back and the piece of paper was passed over my head between the two doctors and the nurse. No one said anything to me. They didn't have to. Ironically, the lump I felt was benign, but between my ribs and chest wall is where my cancer had tucked itself away, only to be discovered when the surgeon put his hand inside to feel around. It is no wonder the procedure hurt, as he had to remove the cancer that had become adhered to my ribs, was deep in my chest wall, infringing on my muscle, and why I was feeling the spasms I described. Thankfully, there was no spread of disease and the lymph node dissection revealed only one macro- and one microscopic node.

My age was the blinding factor in my diagnosis and the genomic story that would become relevant to me later in life had not yet been revealed. It was an incredibly lonely time; the cancer support and advocacy movement was just emerging. There was absolutely nothing for young adults with cancerit was truly the dark ages for people like me. At the time, medical oncologists found my "notion" of starting an organization specifically for this age group of $17-40$ as both curious and unnecessary. The idea of even assigning a distinction to the unique clinical and psychosocial needs of "young adults with cancer" seemed rather "senseless" as it was "too rare of an occurrence." For a time I did visit a support group, but found that I couldn't relate to anything. I was 20 years younger than the next youngest person, with my life experience still pending and miles removed. 
I ultimately found a medical oncologist progressive in his clinical approach who offered a protocol still two years from becoming the standard of care for that time, cyclophosphamide, methotrexate, and fluorouracil (CMF). Given what is known about my tumor profile today, I would be considered seriously undertreated and certainly, Adriamycin, a taxane, and Herceptin, even tamoxifen, would now be included with my orders. That said, I got off rather easy; I'll never know if the error that accidently gave me higher than planned doses of radiation therapy may have inadvertently contributed to my excellent recovery from breast cancer.

When the BRCA1 mutation was discovered in 1994, I was among the early clinical trial participants to be tested. It was no surprise when I tested positive for $B C R A 1$, as did my sister. My father was negative, but we had already assumed it was maternally inherited as it also explained the nature of both my mother's and grandmother's deaths at the age that I am now.

Twenty years after breast cancer, I had a prophylactic salpingo-oophorectomy with a gynecologic oncologist. It was intended as an affirmation of life and ongoing health, so we were all surprised by the incidental finding of ovarian cancer. Luckily, it was well maintained and isolated to the left ovary. But that is where my story diverts from the AYA path, and while no longer a young adult with cancer, today's new technology and how it has influenced me as a YA survivor is incredibly relevant for the younger generation of cancer survivors. While I have never developed bulky ovarian cancer or serious disease spread, over the last eight years, unlike with my breast cancer, I have endured five unique recurrent or new episodes with a disease presentation that is extraordinarily rare, and many of the same feelings I had as a young adult with cancer are present still today.

When I founded it the same year I was diagnosed nearly 30 years ago, Vital Options: Support For Young Adults With Cancer (www.vitaloptions.org) was the first organization of its kind. I was laughed at, and was told I would not succeed and that we would need a parenting organization, which sounded so absurd to me since that was one of the very issues that a young adult deals with. Being in Los Angeles made it easy to rally the entertainment industry and events such as the annual Dance For Life ${ }^{\circledR}$ put us on the map and gave credence to "young adults with cancer," a phrase Vital Options coined at the time.
Today there is a vibrant and well-defined young adult oncology network. But the focus of my early Vital Options efforts was just to get young adults with cancer recognized as a separate and distinct patient population, to give us a forum and a voice, to put faces with diseases, to have us taken seriously, and to stop delayed diagnoses because a patient is believed to be "too young for cancer." My life's work now focuses on cancer media and communications.

The thing about being a young adult is that you don't stay that way-that is, if you survive, which I have somehow managed to do, now as a six-time cancer survivor. Vital Options remains active in the young adult movement and is behind the inception of the annual National Young Adult Cancer Awareness Week ${ }^{\circledR}$ (celebrating its tenth year next April), along with my own involvement in various leadership capacities with organizations and initiatives in the United States and Europe. I would enjoy interacting more with today's young adult survivors, but if you are over 40, then you probably know that there comes a divide. On occasion, I have the gift of hearing from one of the survivor participants from Vital Options' original support groups or TeleSupport ${ }^{\circledR}$ services. Now well into their years, like me they still identify with the feelings of being a young adult with cancer. I still believe that we-as the longest young adult cancer-surviving generation-have something valuable to share. I am enormously proud that my vision now lives on in bigger and more powerful ways than could have been originally imagined.

I have experienced eras in treatment: chemotherapy before there was supportive care, chemotherapy with supportive care, targeted therapy, and I will likely experience yet a new emerging compound known as a PARP inhibitor. My pathology review is no longer limited to a microscope; molecular pathology now genotypes my tissue and makes comparative studies to further define my cancer. I stress these eras in treatment to help highlight my journey and the rather long life I have lived given what we did not know in 1983, and also with the hope that it will positively influence the lives of today's young adults with cancer.

Selma Schimmel, now 56, is the founder and CEO of Vital Options International, a global cancer communications organization that works with key opinion leaders to develop innovative media content to reach and teach about cancer. 


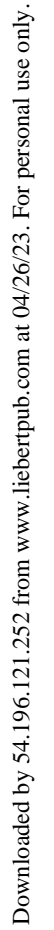

